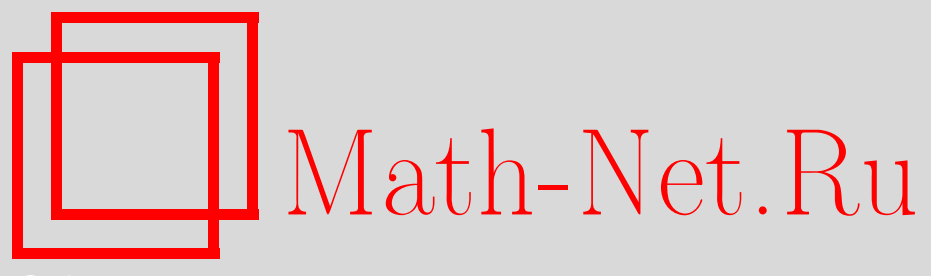

В. З. Гринес, Ю. А. Левченко, О. В. Починка, О топологической классификации структурно устойчивых 3-диффеоморфизмов с двумерными базисными множествами, Матем. заметки, 2015, том 97, выпуск 2, 318 320

DOI: https://doi.org/10.4213/mzm10574

Использование Общероссийского математического портала Math-Net.Ru подразумевает, что вы прочитали и согласны с пользовательским соглашением http: //www . mathnet.ru/rus/agreement

Параметры загрузки:

IP : 54.224 .135 .184

26 апреля 2023 г., 12:39:01 


\section{О топологической классификации структурно устойчивых 3 -диффеоморфизмов с двумерными базисными множествами}

\section{В. З. Гринес, Ю. А. Левченко, О.В.Починка}

В работе рассматриваются диффеоморфизмы, заданные на гладком замкнутом ориентируемом 3-многообразии $M^{3}$ и удовлетворяющие аксиоме $A$ Смейла ( $A$-диффеоморфизмы). Согласно спектральной теореме Смейла [1], неблуждающее множество $N W(f) A$-диффеоморфизма $f$ представляется в виде объединения конечного числа попарно непересекающихся замкнутых инвариантных множеств, называемых базисными, каждое из которых содержит всюду плотную траекторию. Условие нульмерности или одномерности базисного множества $A$-диффеоморфизма $f: M^{3} \rightarrow M^{3}$ не накладывает ограничений на топологию объемлющего многообразия. В случае, когда $N W(f)$ содержит базисное множество $\mathcal{B}$ размерности больше единицы, это не так. Если $\operatorname{dim} \mathcal{B}=3$, то $f$ является диффеоморфизмом Аносова, многообразие $M^{3}$ диффеоморфно трехмерному тору $\mathbb{T}^{3}$, и топологическая классификация таких диффеоморфизмов была получена Фрэнксом и Ньюхаусом в [2], [3]. Если $\operatorname{dim} \mathcal{B}=2$, то, согласно [4], $\mathcal{B}$ является либо аттрактором, либо репеллером. В этом случае топология объемлющего многообразия зависит от топологической структуры множества $\mathcal{B}$.

Напомним, что базисное множество $\mathcal{B}$ диффеоморфизма $f$ называется aттрактором, если существует замкнутая окрестность $U$ множества $\mathcal{B}$ такая, что

$$
f(U) \subset \operatorname{int} U, \quad \bigcap_{j \geqslant 0} f^{j}(U)=\mathcal{B} .
$$

Аттрактор для диффеоморфизма $f^{-1}$ называется репеллером диффеоморфизма $f$. Согласно [5] аттрактор $\mathcal{B}$ диффеоморфизма $f$ называется растягивающимся, если топологическая размерность $\operatorname{dim} \mathcal{B}$ равна размерности неустойчивого многообразия $W_{x}^{u}$ для любой точки $x \in \mathcal{B}$. Сжимающийся репеллер диффеоморфизма $f$ определяется как растягивающийся аттрактор для $f^{-1}$. Согласно [6] базисное множество $\mathcal{B}$ диффеоморфизма $f: M^{3} \rightarrow M^{3}$ называется поверхностным, если оно принадлежит $f$-инвариантной замкнутой поверхности $M_{\mathcal{B}}^{2}$ (не обязательно связной), топологически вложенной в многообразие $M^{3}$ и называемой носителем множества $\mathcal{B}$.

Из работы [7] следует, что любой двумерный аттрактор (репеллер) $A$-диффеоморфизма $f: M^{3} \rightarrow M^{3}$ является либо растягивающимся аттрактором (сжимающимся репеллером), либо поверхностным аттрактором (поверхностным репеллером). Из [8] и [9] следует, что любое многообразие $M^{3}$, допускающее структурно устойчивый диффеоморфизм $f: M^{3} \rightarrow M^{3}$ с двумерным растягивающимся аттрактором (сжимающимся репеллером), диффеоморфно трехмерному тору $\mathbb{T}^{3}$, и, более того, $f$ топологически сопряжен с диффеоморфизмом, полученным из диффеоморфизма Аносова с помощью обобщенной хирургической операции Смейла.

В настоящей работе рассматривается класс $G$, состоящий из сохраняющих ориентацию $A$-диффеоморфизмов $f: M^{3} \rightarrow M^{3}$, неблуждающее множество которых состоит только из двумерных поверхностных базисных множеств и для любого базисного множества $\mathcal{B}$ периода $k$ ограничение диффеоморфизма $f^{k}$ на его периодическую компоненту сохраняет ее ориентацию. Напомним, что базисное множество $\mathcal{B}$ представляется в виде объединения $\mathcal{B}_{1} \cup \cdots \cup \mathcal{B}_{k}, k \geqslant 1$ замкнутых подмножеств таких, что

$$
f^{k}\left(\mathcal{B}_{i}\right)=\mathcal{B}_{i}, \quad f\left(\mathcal{B}_{i}\right)=\mathcal{B}_{i+1}
$$

Работа выполнена при поддержке Российского фонда фундаментальных исследований (гранты №№ 12-01-00672, 13-01-12452-офи-м, 15-01-03687-а) и Российского научного фонда (грант № 14-41-00044).

DOI: $10.4213 / \mathrm{mzm} 10574$ 
$\left(\mathcal{B}_{k+1}=\mathcal{B}_{1}\right)$. Множества $\mathcal{B}_{1}, \ldots, \mathcal{B}_{k}$ называются периодическими компонентами, а число $k$ - периодом базисного множества $\mathcal{B}$.

Обозначим через $\mathcal{A}(\mathcal{R})$ объединение всех аттракторов (репеллеров), принадлежащих $N W(f)$. В силу [10] для любого диффеоморфизма $f \in G$ имеют место следующие утверждения:

1) множества $\mathcal{A}, \mathcal{R}$ не пусты и состоят из одинакового числа $n_{f} \geqslant 1$ базисных множеств;

2) все периодические компоненты базисных множеств имеют один и тот же период $k_{f} \geqslant 1$

3) множество $M^{3} \backslash(\mathcal{A} \cup \mathcal{R})$ состоит из $2 n_{f} k_{f}$ компонент связности, граница каждой из которых состоит в точности из одной периодической компоненты аттрактора и одной периодической компоненты репеллера, а ее замыкание гомеоморфно многообразию $\mathbb{T}^{2} \times[0,1]$.

Из утверждения 3) следует, что многообразие $M^{3}$ гомеоморфно фактор-пространству $M_{\tau}$, полученному из $\mathbb{T}^{2} \times[0,1]$ отождествлением точек $(z, 1)$ и $(\tau(z), 0)$, где $\tau: \mathbb{T}^{2} \rightarrow \mathbb{T}^{2}-$ некоторый гомеоморфизм.

Теорема 1. Пусть многообразие $M^{3}$ допускает диффеоморфизм $f$ из класса $G$. Тогда $M^{3}$ диффеоморфно многообразию $M_{\widehat{J}}$, где $\widehat{J}$ алгебраический автоморфизм тора, заданный матрицей $J$, которая либо является гиперболической, либо совпадает с единичной матрищей

$$
I=\left(\begin{array}{ll}
1 & 0 \\
0 & 1
\end{array}\right)
$$

либо совпадает с матрицей

$$
-I=\left(\begin{array}{cc}
-1 & 0 \\
0 & -1
\end{array}\right)
$$

Представим многообразие $M_{\widehat{J}}$ как пространство орбит $M_{\widehat{J}}=\left(\mathbb{T}^{2} \times \mathbb{R}\right) / \Gamma$, где $\Gamma=\left\{\gamma^{k}\right.$, $k \in \mathbb{Z}\}$ - группа степеней диффеоморфизма $\gamma: \mathbb{T}^{2} \times \mathbb{R} \rightarrow \mathbb{T}^{2} \times \mathbb{R}$, заданного формулой

$$
\gamma(z, r)=(\widehat{J}(z), r-1) .
$$

Обозначим через $p_{\widehat{J}}: \mathbb{T}^{2} \times \mathbb{R} \rightarrow M_{\widehat{J}}$ естественную проекцию.

Введем класс $\Phi \subset G$ модельных диффеоморфизмов. Напомним, что $\operatorname{SL}(2, \mathbb{Z})$ является подмножеством множества $\mathrm{GL}(2, \mathbb{Z})$, состоящим из гиперболических матриц с определителем 1. Пусть $C \in \mathrm{SL}(2, \mathbb{Z})$ - гиперболическая матрица такая, что $C J=J C$. Для $n, k \in \mathbb{N}$ обозначим через $\psi_{n, k}: \mathbb{R} \rightarrow \mathbb{R}$ диффеоморфизм, являющийся сдвигом на единицу времени потока $\dot{r}=\sin 2 \pi n k r$. Для $k=1$ положим $l=0$ и для $k>1$ пусть $l \in\{1, \ldots, k-1\}-$ натуральное число, взаимно простое с $k$. Обозначим через $\chi_{k, l}: \mathbb{R} \rightarrow \mathbb{R}$ диффеоморфизм, заданный формулой

$$
\chi_{k, l}(r)=r-\frac{l}{k} .
$$

Положим $\varphi_{n, k, l}=\psi_{n, k} \chi_{k, l}: \mathbb{R} \rightarrow \mathbb{R}$. Обозначим через $\widetilde{\phi}_{C, n, k, l}: \mathbb{T}^{2} \times \mathbb{R} \rightarrow \mathbb{T}^{2} \times \mathbb{R}$ диффеоморфизм, заданный формулой

$$
\widetilde{\phi}_{C, n, k, l}(z, r)=\left(\widehat{C}(z), \varphi_{n, k, l}(r)\right) .
$$

Непосредственно проверяется, что $\widetilde{\phi}_{C, n, k, l} \gamma=\gamma \widetilde{\phi}_{C, n, k, l}$, откуда следует, что отображение $\phi_{C, n, k, l}: M_{\widehat{J}} \rightarrow M_{\widehat{J}}$, заданное формулой

$$
\phi_{C, n, k, l}=p_{\widehat{J}} \widetilde{\phi}_{C, n, k, l} p_{\widehat{J}}^{-1},
$$

где $p_{\widehat{J}}^{-1}(x)$ полный прообраз точки $x$, является диффеоморфизмом. Обозначим через $\Phi$ множество таких диффеоморфизмов. Из конструкции следует, что любой диффеоморфизм из класса $\Phi$ является структурно устойчивым. 
Tеорема 2. Два диффеоморфизма $\phi_{C, n, k, l}: M_{\widehat{J}} \rightarrow M_{\widehat{J}}, \phi_{C^{\prime}, n^{\prime}, k^{\prime}, l^{\prime}}: M_{\widehat{J^{\prime}}} \rightarrow M_{\widehat{J^{\prime}}}$ uз класса $\Phi$ топологически сопряжены тогда и только тогда, когда

1) существует матрииа $H \in \mathrm{GL}(2, \mathbb{Z})$ такая, что $C H=H C^{\prime}$,

2) $k=k^{\prime}, n=n^{\prime}$ и выполнено хотя бы одно из следующих условий:

- $J H=H J^{\prime} u l=l^{\prime}$,

- $J^{-1} H=H J^{\prime} u k-l=l^{\prime}$,

- $J^{-1} H=H J^{\prime}$ u $l=l^{\prime}=0$.

Напомним, что два диффеоморфизма $f: M^{n} \rightarrow M^{n}, f^{\prime}: M^{\prime n} \rightarrow M^{\prime n}$ называются $\Omega$-сопряженными, если существует гомеоморфизм $h: M^{n} \rightarrow M^{\prime n}$ такой, что

$$
h(N W(f))=N W\left(f^{\prime}\right),\left.\quad h f\right|_{N W(f)}=\left.f^{\prime} h\right|_{N W(f)} .
$$

Теорема 3. Любой дифбеоморфизм из класса $G$ является $\Omega$-сопряженным некоторому диффеоморфизму из класса $\Phi$.

Теорема 4. Любой структурно-устойчивый диффеоморфизм из класса $G$ топологически сопряжен некоторому диффеоморфизму из класса $\Phi$.

\section{СПИСОК ЦИТИРОВАННОЙ ЛИТЕРАТУРЫ}

[1] С. Смейл, УМH, 25:1 (1970), 113-185. [2] J. Franks, Global Analysis, Proc. Symp. in Pure Math., 14, Amer. Math. Soc., Providence, RI, 1970, 61-93. [3] S. E. Newhouse, Amer. J. Math., 92:3 (1970), 761-770. [4] Р. В. Плыкин, Матем. сб., 84:2 (1971), 301-312. [5] R. F. Williams, Inst. Hautes Études Sci. Publ. Math., 43 (1974), 169-203. [6] В. З. Гринес, В. С. Медведев, Е.В. Жужома, Матем. заметки, 78:6 (2005), 813-826. [7] А. W. Brown, J. Mod. Dyn., 4:3 (2010), 517-548. [8] V. Grines, E. Zhuzhoma, Trans. Amer. Math. Soc., 357:2 (2005), 617-667. [9] V. Medvedev, E. Zhuzhoma, J. Dyn. Control Syst., 11:3 (2005), 405-411. [10] В. З. Гринес, В. С. Медведев, Ю. А. Левченко, Журнал СВМО, 12:2 (2010), $7-13$.

\section{В. З. Гринес}

Поступило

Нижегородский государственный

10.01 .2014

университет им. Н. И. Лобачевского

E-mail: vgrines@yandex.ru

Исправленный вариант

27.05 .2014

\section{Ю. А. Левченко}

Нижегородский государственный университет им. Н. И. Лобачевского

E-mail: ulev4enko@gmail.com

\section{О. В. Починка}

Национальный исследовательский университет "Высшая школа экономики"

E-mail: olga-pochinka@yandex.ru 